\title{
Montane Baumschäden durch das Herbizid Trichloressigsäure
}

\author{
- Symptome und mögliche Ursachen
}

\author{
H. Frank, Antje Vincon, J. Reiss \\ Institut für Toxikologie, Universität Tübingen, Wilhelmstraße 56, W-7400 Tübingen
}

\begin{abstract}
Zusammenfassung. Die Ursachen vorzeitiger Nadelverluste bei Koniferen, wie sie in Gebirgswäldern seit mehr als einem Jahrzehnt beobachtet werden, sind noch weitgehend unerkannt.

Es liegt nahe, daß sie durch Luftverunreinigungen oder indirekte atmogene Effekte auf die Bodenchemie ausgelöst werden; doch treten die stärksten Schäden in Regionen auf, die nach üblichen lufthygienischen Standards als relariv wenig belastet gelten. Stärkere Nadeiverluste in größerer Höhenlage deuten auf die Beteiligung böhenabhängiger atmosphären-physikalischer oder -chemischer Faktoren hin, die unter photochemischer Aktivierung primärer anthropogener Luftverunreinigungen zur Bildung von eigentlich phytotoxischen, sekundären Luftschadstoffen führen könnten.

Anthropogene luftgetragene Chlorkohlenwasserstoffe sind hier besonders bedenkenswert. Ihre physikochemischen Eigenschaften, die langjährigen Tendenzen ihrer Emissionen und ImmissionsKonzentrationen, und die Möglichkeit der atmosphären-chemischen Umwandlung in reaktive oder phytotoxische Oxidationsprodukte, insbesondere chlorierte aliphatische Säuren, lassen ihre Beteiligung an der Auslösung montaner Waldschäden wahrscheinlich werden.
\end{abstract}

\section{Problemstellung}

Pigmentausbleichungen und frühzeitige Nadelverluste werden seit ungefähr einem Jahrzehnt an Koniferen in Gebirgswäldern von Industrieländern der nördlichen Hemisphäre beobachtet. Chlorose und Nekrose von Nadeln und Blättern, verminderte Größe und veränderte Form der Blätter von Laubbäumen, Degeneration der Feinwurzeln und der Mykorrhiza sind weitere typische Symptome [1].

Luftverunreinigungen sind an der Auslösung dieser Phänomene wahrscheinlich beteiligt. Schwefeldioxid bei Immissions-Konzentrationen von einigen hundert Mikrogramm pro Kubikmeter ist akut phytotoxisch [2], und hohe Ozonkonzentrationen können zu Chlorose und Nadelverlusten führen [3].

Deposition partikelgebundener toxischer Metalle wie Blei, Zink, Quecksilber und Mobilisierung von Aluminium im Boden in toxischen Konzentrationen durch saure Niederschläge werden als verursachende oder disponierende Faktoren diskutiert $[4,5]$.

Diese Annahmen sind plausibel, doch eine direkte Phytotoxizität in den relevanten Immissions-Konzentrationen konnte bisher experimentell nicht gezeigt werden [6]. Es wird daher postuliert, daß die Schäden durch die synergistische Wirkung mehrerer atmogener "Stress“-Faktoren hervorgerufen werden. Viele atmosphärische Spurenstoffe können beteiligt sein, doch hinreichende analytische Daten gibt es bisher nur für Schwefeldioxid, Stickoxide und Ozon.

Die anthropogenen Emissionen von $\mathrm{SO}_{2}$ und $\mathrm{NO}_{x}$ sind im Laufe dieses Jahrhunderts kräftig gestiegen [7]; doch die Immissions-Konzentrationen in ländlichen Gebieten oder Forsten und ihre langzeirlichen Entwicklungen sind zur geographischen Verbreitung sowie zeitlichen Entwicklung der Schäden nicht korreliert $[8,9]$. Eine bisher unerklärte Tatsache ist weiterhin, daß Bäume in der Nähe von Städten oder Industriezentren z.T. geringere Nadelverluste zeigen als in Regionen, die als Reinluftgebiete bezeichner werden. Die vorwiegend natürliche Spurenkomponente $\mathrm{Ozon}$ schwankt in ihren Immissions-Konzentrationen in der unteren Atmosphäre stark und ist hauptsächlich von Sonneneinstrahlung und stratosphärischen Injektionen abhängig; u.a. entsteht es bei der Photooxidation anthropogener und natürlicher Koblenwasserstoffe (photochemischer Smog, Sommersmog, ,Los Angeles Smog'). Es gibt Hinweise auf langzeitliche Anstiege, doch sind die Schwankungsbreiten der monatlichen oder jährlichen Mittelwerte und die statistische Signifikanz der Trendberechnungen gering [10]. Immissions-Konzentrationen in nicht belasteten Gebirgsregionen können ähnliche Höhen wie in der Nähe von Städten und Industriezentren erreichen $[11,12]$.

Pflanzliche Organismen waren in ihrer natürlichen Umwelt also schon immer zeitweilig hohen Ozonkonzentrationen ausgesetzt; warum sie seit einiger Zeit gegenüber diesem natürlichen atmosphärischen Spurenbestandteil besonders empfindlich geworden sein könnten, ist unbekannt. Andere Vertreter der Gruppe der Photooxidantien, zu denen das Ozon als Leitsubstanz gehört, sind bisher nur sporadisch bestimmt worden, und über ihre phytotoxischen Wirkungen ist wenig bekannt [13] (s.a. $\rightarrow$ S. 215).

Montane Waldschäden scheinen von den jeweiligen geologischen oder bodenchemischen Gegebenheiten nur wenig beeinflußt zu werden, doch ist plausibel, daß Schadwirkungen an Bäumen durch disponierende Faktoren wie Mangel an essentiellen Elementen oder ungenügende Wasserversorgung noch verstärkt werden.

Die Beobachtung ausgedehnter Nadelverluste in Gebirgsre- 
gionen Mitteleuropas mit relativ reiner Atmosphäre und die zeitgleiche Entwicklung in Nordamerika lassen vermuten, $\mathrm{daß}$ die Baumschäden durch anthropogene Luftverunreinigungen oder deren atmosphärische Abbauprodukte ausgelöst werden, die erst seit jüngerer Zeit in großen Mengen emittiert und jetzt weitverbreitet sind $[14,15]$. Dabei könnten solche Verbindungen besondere Bedeutung haben, denen sich Pflanzen im Laufe der Evolution nicht haben anpassen müssen, die also ausschließlich oder hauptsächlich anthropogenen Ursprungs sind und in den betroffenen Gebirgsregionen in Konzentrationen vorliegen, die zur Aufnahme in phytotoxischen Dosen führen könnten.

Für toxikologische Überlegungen bedeutsam sind die vielfachen Möglichkeiten ihrer biotischen oder abiotischen Umwandlung zu reaktiven Intermediaten [16]. Die Höhenabhängigkeit der Nadelverluste [17] könnte z.B. durch intensivere Sonnenstrahlung mit energiereicher Spektralverteilung und die dadurch erhöhte atmosphärische $\mathrm{OH}$ Konzentration erklärt werden, wodurch es zur beschleunigten Bildung sekundärer toxischer Luftverunreinigungen kommen kann $[18,19]$.

Besondere Aufmerksamkeit sollte solchen anthropogenen Verbindungen gewidmet werden,

- die in den beiden letzten Jahrzehnten in besonders stark steigenden Mengen immittiert worden sind,

- die ausreichende atmosphärische Stabilität zur weiträumigen Verfrachtung haben,

- die andererseits durch atmosphären-chemische Reaktionen zu phytotoxischen Produkten aktiviert werden können,

- deren vermutete phytotoxische Umwandlungsprodukte in den betroffenen Gebirgswäldern in erhöhten Konzentrationen vorhanden sind, denn toxikologische Dosis/Wirkungs-Prinzipien sollten auch hier gelten; gewöhnlich sind biologische Wirkungen von der Dosis eines Fremdstoffes in logarithmischer Weise abhängig [20]. Demgemäß sollten Nadelverluste zu geometrischen Anstiegen vermuteter phytotoxischer Fremdstoffe korreliert sein.

\section{Luftgetragene Chlorkohlenwasserstoffe}

Chlorkohlenwasserstoffe erfüllen fast alle genannten Bedingungen. Die ubiquitären anthropogenen $\mathrm{C}_{1}$ - und $\mathrm{C}_{2}$-Halogenkohlenwasserstoffe haben zwar relativ niedrige Säugetiertoxizität, aber Chloroform, Tetrachlormethan, Trichlorethen und Tetrachlorethen sind möglicherweise krebserregend [21]. Ihre Flüchtigkeit und entsprechend kleinen Henry-Konstanten führen dazu, daß sie nach Freisetzung in die Umwelt zu mehr als $99 \%$ im Kompartiment der Atmosphäre bleiben [22].

Die perhalogenierten Koblenwasserstoffe steigen wegen ihrer langen Lebensdauern in ihren atmosphärischen Hintergrund-Konzentrationen noch immer an [23] ( $\rightarrow$ Tabelle).

Die kumulativen Häufigkeitsverteilungen aktueller Konzentrationsbestimmungen der $\mathrm{C}_{2}$-Chlorkohlenwasserstoffe in der bodennahen Atmosphäre von Waldregionen Süddeutschlands zeigen, daß fast alle in höheren ImmissionsKonzentrationen auftreten als in wirklichen Reinluftgebieten; in Städten sind sie erwartungsgemäß noch höher $(\rightarrow A b b .1)$ :

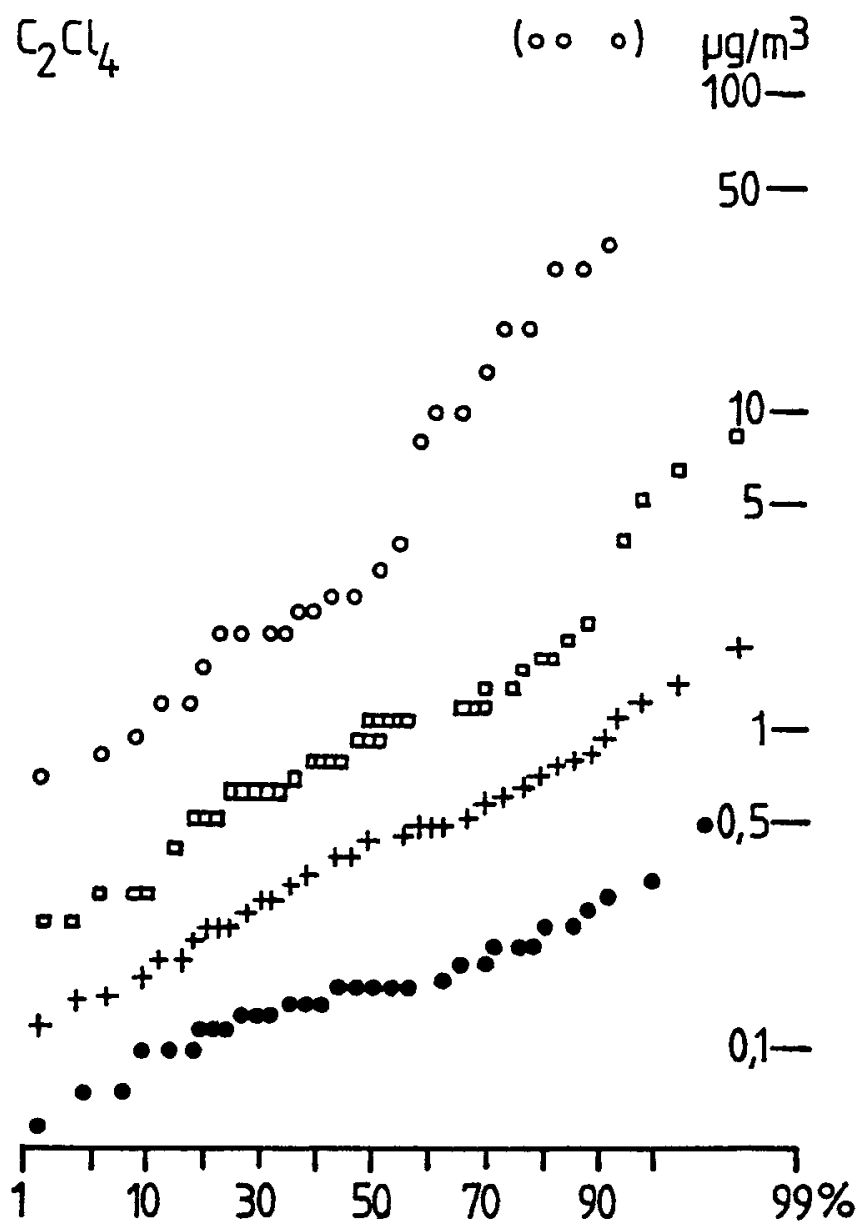

Abb. 1: Vergleichende kumulative Häufigkeitsverteilung der Immissions-Konzentrationen von Tetrachlorethen in der bodennahen Atmosphäre in Tübingen (O), im Nordschwarzwald ( $\square$ ), an der portugiesischen Atlantikküste ca. $15 \mathrm{~km}$ südlich von Lissabon (+), und auf Madeira (•). Die drei höchsten Werte für Tetrachlorethen lagen oberhalb des dynamischen Bereiches des verwendeten Detektors $\left(>100 \mu \mathrm{g} / \mathrm{m}^{3}\right)$

Wegen ihrer mittleren Lebensdauer von Tagen bis Monaten sind die globalen Hintergrundkonzentrationen der Chlorethene zwar relativ gering, doch hat Tetrachlorethen auf der nördlichen Hemisphäre in den Breiten der IndustrieNationen die höchsten Konzentrationen [24]. In ländlichen Gebieten Mitteleuropas schwanken ihre Immissions-Konzentrationen sehr stark abhängig von meteorologischen Bedingungen, Windrichtung und Lage des betreffenden Meßstandortes relativ zu Quellen und Depots. Das atmosphärisch persistente Lösemittel 1,1,1-Trichlorethan allerdings ist im vergangenen Jahrzehnt in seiner globalen Hintergrundkonzentration kräftig gestiegen.

Eine retrospektive Abschätzung der Entwicklung der repräsentativen Immissions-Konzentrationen ist schwierig, da kontinuierliche Messungen an mitteleuropäischen Standorten nicht durchgeführt worden sind. Es ist aber dennoch anzunehmen, daß die mittleren troposphärischen Konzentrationen der $\mathrm{C}_{2}$-Chlorkohlenwasserstoffe seit ihrer Einführung als Entfetter und Lösemittel auch in industriefer- 
Tabelle: Ubiquitäre luftgetragene Halogenkohlenwasserstoffe

\begin{tabular}{|c|c|c|c|c|}
\hline Verbindung & $\begin{array}{r}\text { Weltproduktion } \\
\qquad 10^{3} \mathrm{t} / \mathrm{a}\end{array}$ & $\begin{array}{l}\text { Emission } \\
\qquad 10^{3} \mathrm{t} / \mathrm{a}\end{array}$ & $\begin{array}{r}\text { Hintergrund- } \\
\text { Konzentration } \\
\text { pptv (1987) }\end{array}$ & $\begin{array}{r}\text { Atmosphär. } \\
\text { Lebensdauer } \\
\text { a }\end{array}$ \\
\hline $\begin{array}{l}\text { Chlormethan, Methylchlorid, } \\
\mathrm{CH}_{3} \mathrm{Cl}\end{array}$ & natürlich & $1000-9000$ & 620 & $2-3$ \\
\hline $\begin{array}{l}\text { Brommethan, Methylbromid, } \\
\mathrm{CH}_{3} \mathrm{Br}\end{array}$ & natürlich & & 15 & 2 \\
\hline $\begin{array}{l}\text { lodmethan, Methyliodid, } \\
\mathrm{CH}_{3} \mathrm{I}\end{array}$ & natürlich & 40000 & 1 & 0,01 \\
\hline $\begin{array}{l}\text { Dichlordifluormethan (R 12), } \\
\mathrm{CF}_{2} \mathrm{Cl}_{2}\end{array}$ & 570 & 350 & 400 & $100-170$ \\
\hline Trichlorfluormethan (R 11), & 485 & 280 & 210 & $55-90$ \\
\hline $\begin{array}{l}\mathrm{CFCl}_{3} \\
\text { 1,1,2-Trichlor-1,2,2-trifluorethan (R 113), } \\
\mathrm{C}_{2} \mathrm{Cl}_{3} \mathrm{~F}_{3}\end{array}$ & & 90 & 100 & $60-120$ \\
\hline $\begin{array}{l}\text { Tetrachlormethan, Tetrachlorkohlenstoff, } \\
\mathrm{CCl}_{4}\end{array}$ & 1000 & 110 & 130 & 50 \\
\hline $\begin{array}{l}\text { Trichlormethan, Chloroform, } \\
\mathrm{CHCl}_{3}\end{array}$ & 250 & 20 & 30 & $0,3-0,6$ \\
\hline $\begin{array}{l}\text { Dichlormethan, Methylenchlorid, } \\
\mathrm{CH}_{2} \mathrm{Cl}_{2}\end{array}$ & 500 & 360 & 30 & 0,5 \\
\hline $\begin{array}{l}\text { 1,1,1-Trichlorethan, Methylchloroform, } \\
\mathrm{CH}_{3} \mathrm{CCl}_{3}\end{array}$ & 600 & 520 & 150 & 6 \\
\hline $\begin{array}{l}\text { Tetrachlorethen, Perchlorethylen, } \\
\mathrm{C}_{2} \mathrm{Cl}_{4}\end{array}$ & 1100 & 460 & $20-25$ & 0,4 \\
\hline $\begin{array}{l}\text { Trichlorethen, Trichlorethylen, } \\
\mathrm{C}_{2} \mathrm{HCl}_{3}\end{array}$ & 600 & 600 & 8 & 0,02 \\
\hline
\end{tabular}

Werte aus Ref. 22, 23

nen Regionen gestiegen sind, denn für Tetrachlorethen hat sich die Produktion in der (ehemaligen) Bundesrepublik Deutschland seit 1965 mehr als verdoppelt [25]. Da natürliche Quellen unbekannt sind, sollten sie in anthropogen unbelasteter Atmosphäre nicht vorhanden sein. Vergleichende Messungen an der Atlantikküste Portugals oder auf Madeira [26] zeigen die relativ hohe Belastung mitteleuropäischer Gebirgsregionen (vgl. $\rightarrow$ Abb. 1).

Die Konzentrationen der Halogenkohlenwasserstoffe in den oberen Schichten nicht direkt kontaminierter Böden stehen mit denen in der Atmosphäre im Gleichgewicht. Bemerkenswert ist die Tatsache, daß Chloroform in der Bodenluft mancher Gebirgswälder in höheren Konzentrationen auftritt, als bei Gleichgewicht zu erwarten wäre [27].

\subsection{Photooxidation luftgetragener $\mathrm{C}_{2}$-Chlorkohlenwas- serstoffe}

$\mathrm{C}_{2}$-Chlorkohlenwasserstoffe werden gemäß ihrer hohen Lipophilie von Lipiden und Wachsen rasch aufgenommen [28]. Wenn Koniferen oder Laubbäume dem Lösemittel Tetrachlorethen in Konzentrationen ausgesetzt werden, die ungefähr zehnfach über den durchschnittlichen ImmissionsKonzentrationen in süddeutschen Waldgebieten liegen, werden bei gleichzeitiger intensiver Sonnenbestrahlung photosynthetische Pigmente zerstört und Nekrotisierung der Blätter von Laubbäumen beobachtet [29]. Modellexpe- rimente bestätigen, daß Chlorophyll $A$ und $\beta$-Carotin besonders empfindlich sind; andere Halogenkohlenwasserstoffe haben ähnliche Effekte [30, 31]. Wie erwartet, ist die Pigmentausbleichung bei kürzeren Wellenlängen rascher [32]. KOLBE berichtete bereits 1845 , daß bei Photooxidation von Tetrachlorethen Trichloressigsäure (TCA) entsteht [33]. In der Gasphase wird Tetrachlorethen leicht und in hoher Ausbeute (80\%) zu Tetrachloroxiran und Trichloressigsäurechlorid photooxidiert [34]. (Hydroxyl-Radikale, die wichtigsten atmosphären-chemischen Oxidantien, sind wahrscheinlich auch beim troposphärischen Abbau von $\mathrm{C}_{2}$-Chlorkohlenwasserstoffen beteiligt, wie die Bildung von Formylchlorid und Phosgen nagelegt [35]. Umsetzung mit Ozon, die bei sterisch gehinderten Olefinen zum Epoxid führt, ist wahrscheinlich zu langsam [36]). Auch in kondensierter Phase führt die Photooxidation von Tetrachlorethen zu Tetrachloroxiran [37] (I).

Die beiden anderen $\mathrm{C}_{2}$-Chlorkohlenwasserstoffe, die in der freien Atmosphäre auftreten, können ebenfalls zu TCA abgebaut werden: Oxidation von 1,1,1-Trichlorethan führt in mehreren Stufen zum Trichloracetaldehyd (Chloral), welches leicht zu TCA weiteroxidiert werden kann(II), ebenso wie die Epoxidation von Trichlorethen und anschließende Umlagerung unter Wanderung eines Chloratoms (III): 


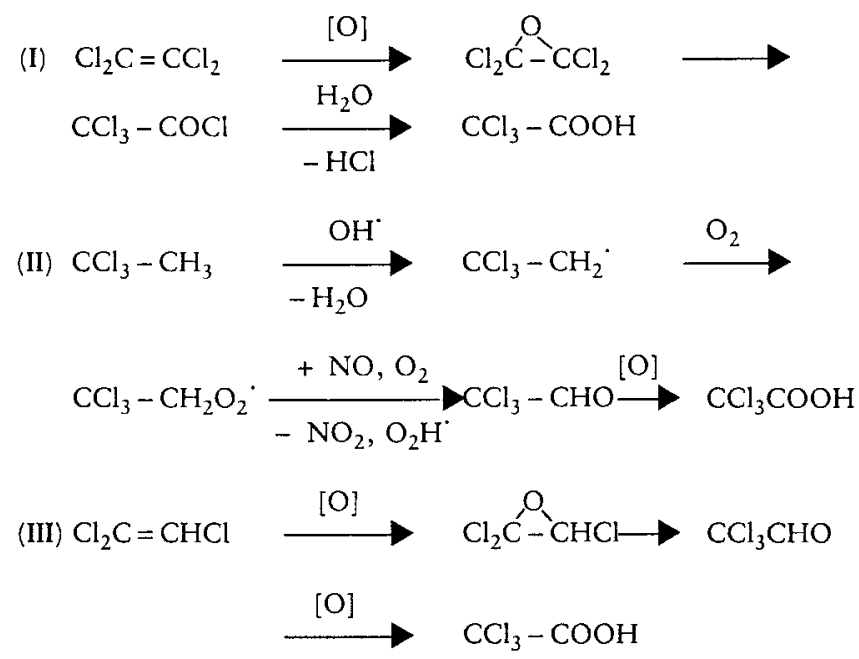

TCA wurde bereits in den siebziger Jahren als atmosphären-chemisches Abbauprodukt von Tetrachlorethen diskutiert [38, 39], konnte aber in der Umwelt nicht nachgewiesen werden. Bei gezielter Optimierung der Spurenanalytik gelingt es, TCA im Boden und in Nadelproben aus relativ unbelasteten Gebirgswäldern zu bestimmen [40]. Auch das homologe Oxidationsprodukt des Trichlorethens, die unter Wasserstoff-Umlagerung (statt Chlor-Umlagerung wie bei Formelschema II) entstehende Dichloressigsäure, ist in Regenwasser-Proben gefunden worden [41] (IV):

$$
\begin{aligned}
& \text { (IV) } \mathrm{Cl}_{2} \mathrm{C}=\mathrm{CHCL} \stackrel{[\mathrm{O}]}{\longrightarrow} \mathrm{Cl}_{2} \stackrel{\mathrm{C}-\mathrm{O}}{\mathrm{CHCl}} \rightarrow \mathrm{CHCl}_{2}-\mathrm{COCl} \\
& \stackrel{-\mathrm{HCl}}{\stackrel{\mathrm{H}_{2} \mathrm{O}}{\longrightarrow}} \mathrm{CHCl}_{2}-\mathrm{COOH}
\end{aligned}
$$

Unter Annahme einer jährlichen Emission von ca. $75000 \mathrm{t}$ Tetrachlorethen (ehemalige Bundesrepublik) und bei einem Umsatz in ähnlicher Höhe wie bei Photooxidation im Labor $(80 \%)$ kann durch troposphärische Oxidation die Entstehung von $60000 \mathrm{t}$ Trichloressigsäure erwartet werden. Das jährlich verbrauchte und freigesetzte 1,1,1-Trichloressigsäure erwartet werden. Das jährich verbrauchte und freigesetzte 1,1,1-Trichlorethan (50000 t) trägt durch $\mathrm{OH}^{-}$-abhängige Oxidation mit wahrscheinlich noch höherer prozentualer Ausbeute ebenfalls zur Bildung von Trichloressigsäure bei. Dabei dürfte auf Grund der unterschiedlichen atmosphärischen Stabilitäten der erstere Chlorkohlenwasserstoff eher zu regionaler, der zweite zu global verteilter Deposition von Trichloressigsäure führen. Abb. 2 + 3 zeigen TCA-Konzentrationen während des Sommers 1989 in Nadeln zweier Fichten von Beobachtungsflächen mit unterschiedlichen Nadelverlusten.

Die durchschnittlichen Nadelverluste im Frühjahr 1989 lagen bei $32 \%$ bzw. $20 \%$, dem niedrigsten Wert in BadenWürttemberg. Im Laufe des Sommers 1989 stiegen die TCA-Gehalte bis September/Oktober stetig an (besonders bei der Beobachtungsfläche mit stärkeren Nadelverlusten), im Laufe des Spätherbstes und Frühwinters sanken sie allmählich wieder.

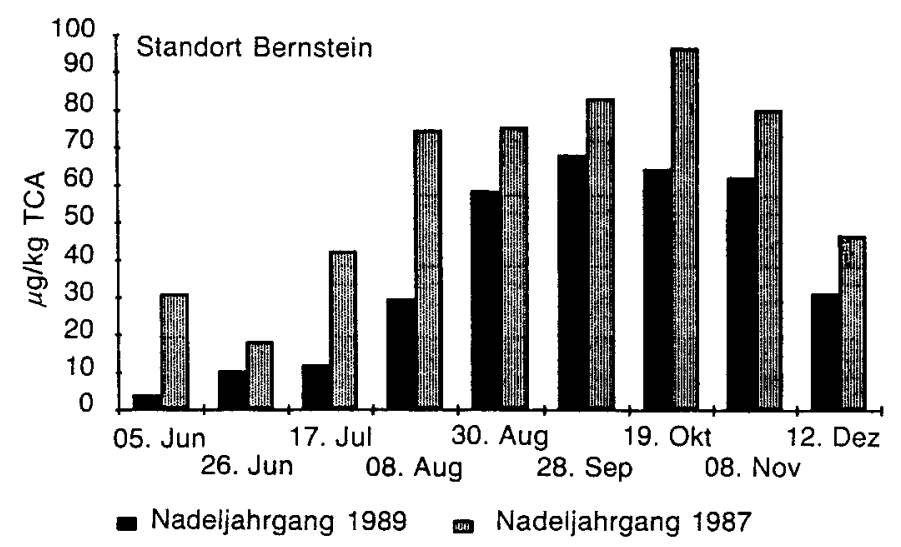

Abb. 2: Trichloressigsäure in Nadeln einer Fichte auf der Beobachtungsfläche $55 \mathrm{~A}$ (Bernstein, Forstamt Rotenfels, Nordschwarzwald) im Laufe des Sommers 1989. Durchschnittlicher Nadelverlust im Frühjahr 1989: $32 \%$

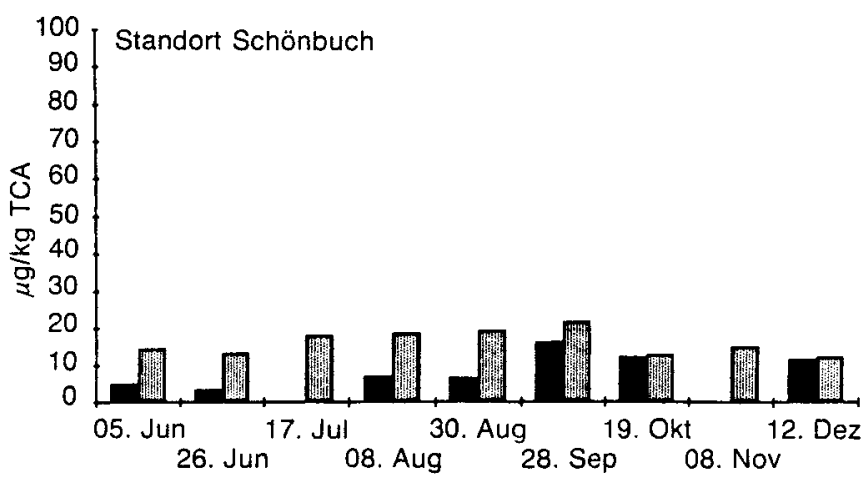

Nadeljahrgang 89 madeljahrgang 87

Abb. 3: Trichloressigsäure in Nadeln einer Fichte auf der Beobachtungsfläche $71 \mathrm{~A}$ (Schönbuch, Forstamt Bebenhausen) im Laufe des Sommers 1989. Durchschnittlicher Nadelverlust im Frühjahr 1989: $20 \%$

Nadeln des Jahrgangs 1987 wiesen höhere TCA-Konzentrationen auf als die am gleichen $Z$ weig wachsenden jüngsten Nadeln. Auch bei Buchenblättern wurde ein ähnlicher jahreszeitlicher Verlauf beobachtet $(\rightarrow A b b .4)$ :

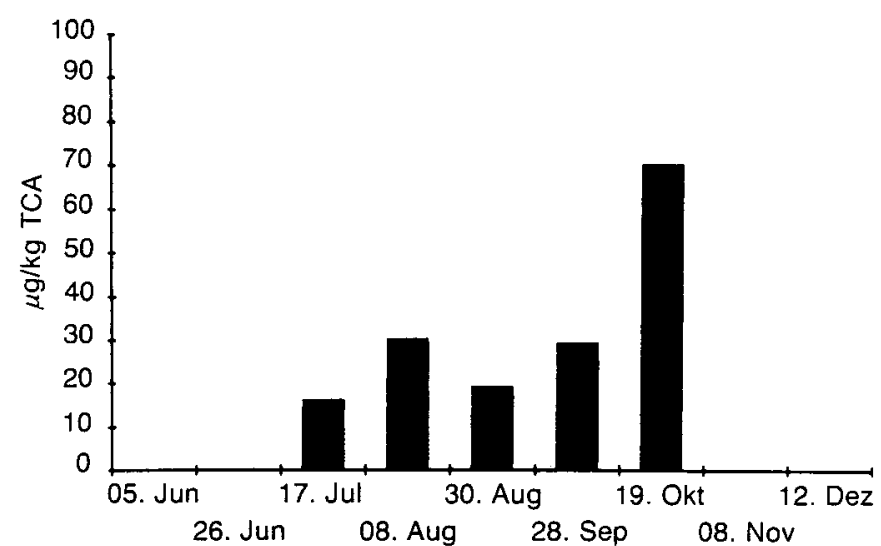

Abb. 4: Trichloressigsäure in Blättern einer Buche (Fagus silvatica) im Laufe des Sommers 1989 
Atmosphärische Spurenkomponenten, die wahrscheinlich ebenfalls aus $\mathrm{C}_{2}$-Chlorkohlenwasserstoffen entstehen, sind Chloroform und Tetrachlormethan.

Chloroform wird in ländlichen Gebieten in ImmissionsKonzentrationen bis $1 \mu \mathrm{g} / \mathrm{m}^{3}$ gefunden, obwohl es kurze atmosphärische Lebensdauer hat und kaum noch als Lösemittel eingesetzt wird; das letztere gilt auch für Tetrachlormethan, dessen globale Hintergrundkonzentration noch immer steigt [23]. Da die direkten Emissionen gering sind, werden natürliche oder indirekte anthropogene Quellen vermutet. Decarbonylierung von Trichloracetylchlorid und Dichloracetylchlorid (V) sowie Decarboxylierung von TCA (VI) sind mögliche Quellen für diese beiden $\mathrm{C}_{1}$-Chlorkohlenwasserstoffe $[42,43]$ :

$$
\begin{aligned}
& \mathrm{CCl}_{3}-\mathrm{COCl} \longrightarrow \mathrm{CCl}_{4}+\mathrm{CO} \\
& \mathrm{CHCl}_{2} \mathrm{COCl} \longrightarrow \mathrm{CHCl}_{3}+\mathrm{CO}
\end{aligned}
$$

Reaktion VII trägt wahrscheinlich zu den relativ hohen Mengen von Chloroform im Waldboden mancher Gebirgswälder bei. TCA konnte in zeitweise hohen, aber stark schwankenden Konzentrationen im Boden nachgewiesen werden $[44,45]$.

(VII)

$$
\mathrm{CCl}_{3}-\mathrm{COOH} \longrightarrow \mathrm{CHCl}_{3}+\mathrm{CO}_{2}
$$

\section{Trichloressigsäure}

\section{- Ein ubiquitäres Herbizid}

Trichloressigsäure (TCA) wurde vor mehr als vierzig Jahren als Herbizid gegen einkeimblättrige Unkräuter eingeführt [46]. Chlorose und Nekrose sonnenbeschienener Blätter sind typische phytotoxische Wirkungen; außerdem hat sie formative Effekte, die wahrscheinliche auf seine Wirkung auf die Meristem-Zellen zurückzuführen ist [47-50]. Veränderung und Reduktion der Wachsschicht der Kutikel und eine dadurch hervorgerufene höhere Wasserpermeabilität sind ebenfalls bekannt $[51,52]$. Derivate von TCA haben zudem fungizide und bakterizide Wirkung [53]. Die biochemische Ursache der Phytotoxizität scheint in der Hemmung der Pantothensäure-Biosynthese zu liegen [54], doch dies ist nicht gesichert (biochemische Untersuchungen zum Mechanismus der Phytotoxizität wurden nicht weitergeführt, als TCA durch andere Herbizide verdrängt wurde). TCA wird als hygroskopische, gut wasserlösliche Verbindung im Boden durch Niederschläge leicht mobilisiert und ausgewaschen [55].

Ihre akute Phytotoxizität ist hauptsächlich an Nutzpflanzen und Unkräutern mit landwirtschaftlicher Bedeutung unter Applikation relativ hoher einmaliger Dosen untersucht worden; über seine chronischen Wirkungen bei Dauerexposition in Konzentrationen, wie sie in der Umwelt auftreten, ist nichts bekannt. Zur Wirkung an Waldbäumen gibt es keine Untersuchungen, obwohl TCA zeitweise in der Forstwirtschaft eingesetzt worden war.

Die Resorption von TCA beim Diffundieren durch Epicuticular-Wachse und Plasmodesmen ist langsam, da sie im neutralen Bereich vollständig dissoziiert ist. Abb. 5 zeigt die relativ langsame Aufnahme der freien Säure in das Blatt- gewebe; der weitaus größere Teil blieb oberflächlich adsorbiert. Nach Überschreiten des Konzentrationsmaximums nach ca. 2 Wochen fiel der TCA-Gehalt in den Nadeln mit einer Halbwertszeit von etwa 10 Tagen. Ähnliche Metabolisierungs-Raten und/oder Mobilisierungs-Raten sind auch bei anderen Pflanzen ermittelt worden [56].

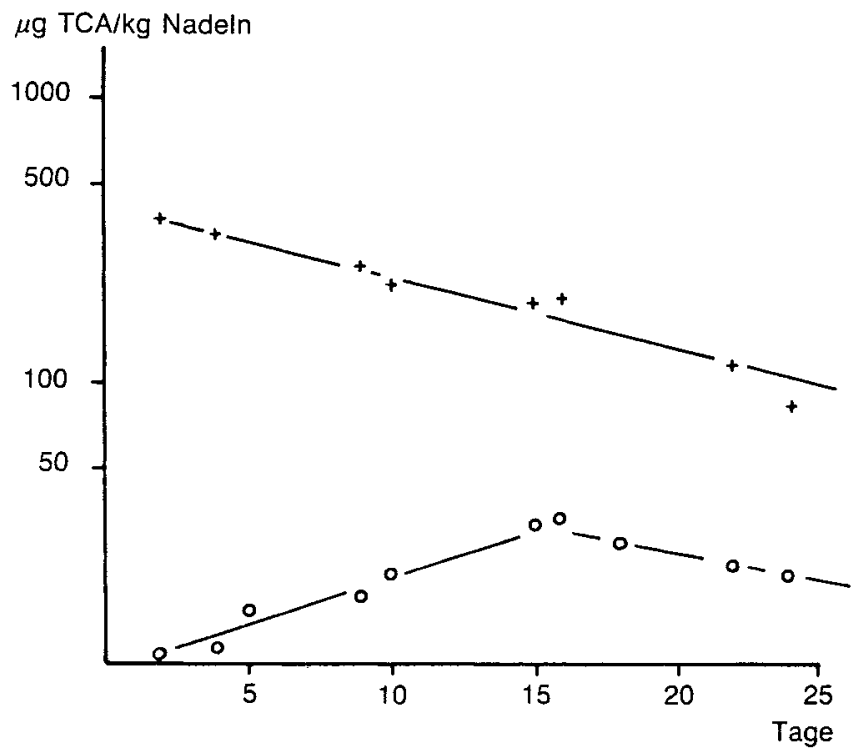

Abb. 5: Trichloressigsäure in Nadeln einer Fichte (Picea abies), die mit einer wäßrigen Lösung von $1,8 \mathrm{mg}$ Trichloressigsäure in $250 \mathrm{ml}$ Wasser besprüht worden war. Obere Linie, GesamtTCA in und auf den Nadeln, untere Linie nach Spülung mit destilliertem Wasser

Bei Fichtennadeln aus dem Freiland befinden sich die „natürlichen" TCA-Mengen fast ausschließlich innerhalb der Nadel. In manchen Fällen sind geringe Anteile von bis zu $10 \%$ mit Wasser abwaschbar, also offenbar auf der Oberfläche adsorbiert. Die in der Nadelmatrix vorhandene TCA könnte entweder als wäßrige Lösung aus dem Boden aufgenommen oder im Gewebe aus $\mathrm{C}_{2}$-Chlorkohlenwasserstoffen durch biotische oder abiotische Oxidation gebildet werden, wobei Blattpigmente als Photosensibilisatoren wirken könnten. Denkbar ist auch eine Deposition aus der Atmosphäre in Form lipophiler Vorläufer, zum Beispiel Tetrachloroxiran oder Trichloressigsäurechlorid.

Da TCA im Blattgewebe metabolisiert oder mobilisiert wird, ist die jeweilige Konzentration vom Gleichgewicht von Bildung/Deposition und Abbau/Mobilisierung abhängig. Daraus folgt, daß sich die Geschwindigkeit der Aufnahme $\left(V_{\text {dep }}\right)$ bei einer Gleichgewichtskonzentration $\left(c_{e q}\right)$ und von $10 \mathrm{ppb}$ und einer Halbwertszeit $\left(\mathrm{t}_{1 / 2}\right)$ von $10 \mathrm{Ta}-$ gen nach

$\mathrm{V}_{\mathrm{dep}}[\mu \mathrm{g} / \mathrm{d} \times \mathrm{kg}]=\mathrm{c}_{\mathrm{eq}} \times \frac{\ln 2}{\mathrm{t}_{1 / 2}}$

als $0,7 \mu \mathrm{g} / \mathrm{kg}$ pro Tag berechnen läßt. Die Unterschiede in den TCA-Spiegeln jüngerer und älterer Nadeln könnten durch einen entsprechend langsameren metabolischen Grundumsatz bei älteren Nadeln ihre Erklärung finden. Aufnahme bis zum Sechsfachen der in der Natur gefundenen Konzentration bei Applikation mit dem Gießwasser 
führt schon nach 3 Monaten zu Chlorophyll-Ausbleichung und massiven Nadelverlusten.

TCA wurde bisher in allen Nadel- und Blattproben identifiziert, die vom Schwarzwald bis zu den Nordalpen gesammelt wurden, wobei die Konzentrationen je nach Jahreszeit und Standort zwischen 10 und 100 ppb variieren; in einzelnen Fällen wurden Konzentrationen bis 150 ppb bestimmt. TCA ist als anthropogene Verunreinigung in der Hydrosphäre schon seit längerer Zeit bekannt; sie entsteht $z$.B. bei der Chlorung huminstoff-haltigen Wassers [57]. Auch in Trinkwasserproben wird sie regelmäßig in Konzentrationen von einigen Mikrogramm pro Liter gefunden [58]. Im Gegensatz zu ihrer hohen Phytotoxizität hat TCA geringe Säugetiertoxizität, und eine tägliche Aufnahme von einigen Mikrogramm ist daher unbedenklich. TCA-Konzentrationen im Blutserum von Arbeitern, die beruflich $\mathrm{C}_{2}$-Chlorkohlenwasserstoff-Dämpfen ausgesetzt sind, können mehr als tausendfach höher sein, ohne daß gesundheitliche Beeinträchtigungen beobachtet werden [59].

Verschiedene Teilaspekte der Wirkungskette von der Aufnahme und Umweltverteilung luftgetragener $\mathrm{C}_{2}$-Chlorkohlenwasserstoffe bis zur Manifestation der Phytotoxizität müssen noch im Detail untersucht werden. Durchgeführt werden sollten u.a.:

- Untersuchungen über die quantitativen Beiträge der denkbaren Bildungswege von TCA,

- Untersuchungen über potentielle synergistische Effekte durch andere atmosphärische Spurenbestandreile wie Ozon oder Stickoxide,

- umfangreiche chronische Expositionsexperimente mit Waldbäumen mit zwei- bis dreifach über den in der Natur gefundenen TCA-Dosen.

\section{Schlußfolgerungen}

Die Hypothese der Beteiligung luftgetragener anthropogener Halogenkohlenwasserstoffe an der Auslösung montaner Waldschäden wird folgendermaßen untermauert:

- Die meisten anthropogenen $C_{1}$ - und $C_{2}$-Halogenkohlenwasserstoffe sind in ihren Immissionen in den letzten Jahrzehnten stark und kontinuierlich gestiegen, sowohl in der Umgebung von Verbrauchern und Emittenten, als auch in sog. Reinluft-Gebieten.

- Ihre hohe Lipophilie und Diffusionsfähigkeit in pflanzliche Wachse und Lipide führt zu rascher Aufnahme und Gleichgewichtseinstellung zwischen Atmosphäre und pflanzlichem Gewebe.

- Alle Halogenkohlenwasserstoffe beschleunigen die lichtabhängige Zerstörung von Blatt-Pigmenten.

- Die in großem Umfang emittierten $C_{2}$-Halogenkohlenwasserstoffe (weltweit mehr als $2 \mathrm{Mill} \mathrm{t/a)} \mathrm{werden} \mathrm{in} \mathrm{der} \mathrm{Atmosphäre} \mathrm{zu} \mathrm{stark}$ phytotoxischen Chloressigsäuren abgebaut.

Luftgetragene Halogenkohlenwasserstoffe sind also auf zweierlei Weise an der Auslösung montaner Waldschäden beteiligt:

1. durch direkte photodynamische Zerstörung von PhotosynthesePigmenten,

2. auf indirektem Wege durch Bildung stark phytotoxischer Chloressigsäuren als Hauptprodukte ihres atmosphärischen Abbaus.

\section{Danksagung:}

Dem Bayerischen Staatsministerium für Landesentwicklung und Umweltfragen und dem Bundesministerium für Forschung und Technologie wird für die finanzielle Unterstützung dieser Arbeiten gedankt.

\section{Literatur}

[1] P. SCHÜTT; E. B. CowLING: Waldsterben, a general decline of forests in Central Europe: Symptoms, development, and possible causes. Plant Disease 69, 548-558 (1985)

[2] A. H. LegGe; D. R. JAQUes; G. W. HarveY; H. R. Krouse; H. M. BROWN; E. C. RHOdES; M. NASAL; H. U. SCHEllHase; J. Mayo; A. P. Hartgerink; P. F. Lester; R. G. AmundSON; R. B. WALKER: Sulphur gas emissions in the Boreal Forest: The West Whitecourt case study. Water Air Pollut. 15, 77-85 (1980)

[3] L. S. Dochinger; C. E. Seliskar: Air pollution and the chlorotic dwarf disease of Eastern white pine. For. Sci. 16, 46-55 (1970)

H. Sandermann; C. Langebartels; W. Heller: Ozonstreß bei Pflanzen. UWSF 2/2,14-15 (1990)

[4] D. Godbold; H. SChlegel; A. HutTermann: Heavy metals a possible factor in spruce decline. Düsseldorf, VDI-Bericht 560, $703-716(1985)$

[5] B. UlRICH: Interaction of indirect and direct effects of air pollutants in forest, in: Air Pollution and Plants. C. TroyANOwSKI (Ed.) $\mathrm{VCH}$, Weinheim 1985 , p. $149-181$

[6] E. B. Cowling: Recent changes in chemical climate and related effects on forests in North America and Europe. Ambio 18, $167-171$ (1989)

[7] G. GsChwandtNer; K. Gschwandtner; K. EldRidGe; C. MANN; D. MOBLEY: Historic emissions of sulfur and nitrogen oxides in the United States from 1900 to 1980. J. Air Poll. Control Assn. 35, 139-149 (1986)

[8] D. Paffrath; W. Peters: Untersuchung groß3räumiger Luftschadstoffbelastungen im Zusammenhang mit den Waldschäden in Bayern DFVLR-Forschungsbericht, Oberpfaffenhofen, West Germany, 1987

[9] Statistisches Landesamt Baden-Württemberg. 1977 - 1989. Statistische Berichte Umwelt, Stuttgart

[10] W. Attmannspacher; R. Hartmannsgruber; P. LANG: Langzeittendenzen des Ozons der Atmosphärc aufgrund der 1967 begonnenen Ozonmeßreihe am Meteorologischen Observatorium Hohenpeißenberg. Meteorol. Rdsch. 37, 193-199 (1984)

[11] H. B. SinCH; F. L. LUDwiG; W. B. JoHnson: Tropospheric ozone: Concentrations and variabilities in clean remote atmospheres. Atmos. Environ. 12, $2185-2: 96$ (1978)

[12] R. G. DER.WENT; P. J. A. KaY: Factors influencing the ground level distribution of ozone in Europe. Environ. Pollut. 55, $191-219(1988)$

[13] R. J. Youngman; E. F. Elstner: Herbizide, in: Pflanzentoxikologie. B. HocK; E. F. ElSTNER (Eds.) BI-Publishers, Mannheim 1984 , p. $132-151$

C. Trojanowski (Ed.): Air Pollution and Plants. VCH Verlagsgesellschaft, Weinheim 1985

[14] H. Frank: Waldschäden durch Photooxidantien? Nachr. Chem. Tech. Lab. 32, $298-305(1984)$

[15] R. Debus; B. Dittrich; P. SChröder; J. Vollmer: Biomonitoring organischer Luftschafstoffe, in: Handbuch des Umweltschutzes et al. J. VoGL (Ed.). Ecomed Landsberg 1989, 45. Erg. Lfg., p. $1-64$

[16] D. HensChler: Mechanismen der Aktivierung chlorierter aliphatischer Verbindungen - experimentelle Zugänge und klinische Bedeutung. Arzneim.-Forsch./Drug Res. 27, 1827 - 1832 (1977)

[17] G. HartmanN; R. Uebel; R.Stock: Zur Verbreitung der Nadelvergilbung an Fichte im Harz. Forst. Holzw. 40, 286- 292 (1985)

[18] P. B. Shepson; T. E.Kleindienst; E. O. Edney; G. Nannie; J. Pittman; L. Cupitt; L. Claxton: The mutagenic activity of irradiated toluene $/ \mathrm{NO}_{\mathrm{x}} / \mathrm{H}_{2} \mathrm{O} /$ air mixtures. Environ. Sci. Technol. $19,620-627$ (1985)

[19] T. E. Kleindienst; P. B. Shepson; E. O. Edney; L. CupitT; L. D. Claxton: The mutagenic activity of the products of propylene photooxidation. Environ. Sci. Technol. 19, 249-255 (1985) 
[20] C. L. BLIss: Some principles of bioassay. Am. Sci. 45, 449-466 (1957)

[21] D. HENSCHLER: Maximale Arbeitsplatzkonzentrationen. VCH, Weinheim 1989

[22] S. PATERsON: Equilibrium models for the initial integration of physical and chemical properties, in: Environmental Exposure from Chemicals, Vol. I. W. B. NeEly; G. E. Blau (Eds.) CRC Press, Boca Raton, 1985 , p. $217-231$

[23] C. R. PenRson: $C_{1}$ and $C_{2}$-Halocarbons, in: Handbook of Environmental Chemistry, Vol. 3B, Anthropogenic Compounds. O. Hutzinger (Ed.) Springer, Berlin 1982, p. $69-88$

P. FABIAN: Halogenated hydrocarbons in the atmosphere, in: Handbook of Environmental Chemistry, Vol. 4A, Air Pollution. O. Hutzinger (Ed.) Springer, Berlin 1984, p. 23-51

J. A. Logan; M. J. Prather; S. C. Wofsy; M. B. MC Elroy: Tropospheric chemistry: a global perspective. J. Geophys. Res. 86, $7210-7254$ (1981)

R. G. PrinN; P. G. Simmonds; R. A. Rasmussen; R. D. RoSen; F. N. Alyea; C. A. Cardelino; A. J. Crawford; D. M. CRUNNOLD;P. J. FRASER; J. E. LOVELOCK: The atmospheric lifetime experiment 1. Introduction, instrumentation, and overview. J. Geophys. Res. 88, 8353-8367 (1983)

[24] H. B. SinGH; L. J. Salas; R. E. Stiles: Selected man-made halogenated chemicals in the air and oceanic environment. J. Geophys. Res. 88, 3675 - 3683 (1983)

[25] Statistisches Bundesamt Wiesbaden. Fachserie 4, Reihe 3.1, Kohlhammer, Stuttgart 1988

[26] H. Frank; W. Frank, H. J. C. Neves: Atmos. Environ 1990, im Druck

[27] H. Frank; W. Frank; D. ThiEl: $C_{1}$ - and $C_{2}$-halocarbons in soil-air of forests. Atmos. Environ. 23, $1333-1335$ (1989)

[28] H. FRANK; W. FRANK: Photochemical activation of chloroethenes leading to destruction of photosynthetic pigments. Experientia 42, $1267-1269$ (1986)

[29] H. FranK; W. FrANK: Chlorophyll-bleaching by atmospheric pollutants and sunlight. A probable cause of the tree damages observed in recent years. Naturwissenschaften 72, 139-141 (1985)

[30] G. Grimmer; W. SCHMidT: Modellversuche zur Phytotoxizität von Halogenkohlenwasserstoffen. Angew. Chem. Intern. Ed. 25, 808-809 (1986)

[31] R. Debus; P. SCHRóder: Wirkung von Halon 1211 (Bromchloridfluormethan) auf Kresse. Düsseldorf, VDI-Bericht 745 , $563-572$ (1989)

[32] R. ERNING: Modeliversuche zur photochemischen Wirkung von chlorierten Kohlenwasserstoffen und anderen organischen Substanzen auf $\beta$-Carotin. Zulassungsarbeit, Fachhochschule Münster 1987

[33] H. KOLBE: Beiträge zur Kenntnis der gepaarten Verbindungen. Ann. Chem. 54, 145-188(1845)

[34] C. SCHOTT; H. J. SCHUHMACHER: Die photochemische Chlorierung und die durch Chlor sensibilisierte photochemische Oxidation von Tetrachloräthylen. Z. Physikal. Chem. 49, 107-125 (1941)

[35] E. C. Tuazon; R. Atkinson; S. M. Aschmann; M. A. Good. MAN; A. M. WINER: Atmospheric reactions of chloroethenes with the $\mathrm{OH}$ radical. Int. J. Chem. Kinet. 20, $241-265$ (1988)

[36] R. AtKInson; W. P. Carter: Kinetics and mechanisms of the gas-phase reactions of ozone with organic compounds under atmospheric conditions. Chem. Rev. 84, $437-470$ (1984)

[37] A. I. TsChernjawski; W. A. POl.UekTOV: Die Oxidationskinetik symmetrischer Dichlorethene. Dokl. Akad. Nauk. SSSR 221, 900-903 (1975)

[38] B. W. Gay; P. L. Hanst; J. J. Bufalini; R. C. Noonan: Atmospheric oxidation of chlorinated ethylenes. Environm. Sci. Technol. 10, 58-67 (1976)
[39] H. B. SiNGH: Phosgene in ambient air. Nature 264, 428-429 (1976)

[40] H. FranK; J. VITAL; W. FranK: Oxidation of airborne $\mathrm{C}_{2}$-chlorocarbons to trichloroacetic and dichloroacetic acid. Fresenius' Z. Anal. Chem. 333, 713 (1989)

[41] G. R. FUCHS; K. BÄCHMANN: Ionen-chromatographische Bestimmung von Chloracetat und Dichloracetat in Niederschlagswasserproben. Fresenius Z. Anal. Chem. 327, 205-212 (1987)

[42] H. B. Singh; D. Lillian; A. Appleby; L. Lobban: Atmospheric formation of carbon tetrachloride from tetrachloroethylene. Environ. Letters 10, 252-256 (1975)

[43] A. Appleby; J. Kazazis; D. Lillian; H. B. Singh: Atmospheric formation of chloroform from trichloroethene. J. Environ. Sci. Health A 11, $711-715$ (1976)

[44] H. Frank: Trichloressigsäure im Boden: Eine Ursache neuartiger Waldschäden. Nachr. Chem. Techn. Lab. 36, 889 (1988)

[45] I. RenNeR; D. Mühlhausen: Immissionsbelastungen Konsequenzen für die Grundwasserqualität. Düsseldorf, VDI-Bericht 745, $467-482$ (1989)

[46] K. C. BARrons; , R. W.Hummer: Basic herbicidal studies with derivatives of TCA. Agric. Chemicals 6, 48-121 (1951)

[47] F. M. Ashton; A. S. Craft: In: Mode of Action of Herbicides. Chapter 8, Aliphatics. Wiley-Interscience, New York 1973, p. $110-125$

[48] P. C. Kearney; C. I. Harris; D. D. Kaufmann; T. J. Sheets: Behaviour and fate of chlorinated aliphatic acids in soils, in: Advances in Pest Control Research, Vol. VI. R. L. Metcalf (Ed.). Wiley-Intersience, New York 1965, p. 1-30

[49] B. ABERG: Plant growth regulators. Swedish J. Agric. Res. 12, $51-61(1982)$

[50] H. ZötTl: Untersuchungen über die Wirkung von Trichloracetaten und anderen Halogenacetaten auf pflanzliches Gewebe. $Z$. Naturforschg. 8b, $317-323$ (1953)

F. MAYER, 1957. Zur Wirkungsweise von trichloracetar auf die höhere Pflanze. Z. Naturforschung 12 b, 336-346 (1957)

[51] F. S. GOOCH; L. W. ERBE: The effects of monochloroacetic acid, dichloroacetic acid and trichloroacetic acid on the tissues of Elodea. Proc. 20th Couthern Weed Conf. 1967, p. 287 O. R. Dewey; P. Gregory; K. Pfelffer: Factors affecting the susceptibility of peas to selective dinitroherbicides. Proc. British Weed Control Conf 3rd Meeting. 1956, p. 313-327

[52] B. E. JUNIPER: The effect of pre-emergent treatment of peas with trichloroacetic acid on the sub-microscopic structure of the leaf surface. New Phytolog. 58, 1-5 (1959)

[53] H. Jansen Van RensburG; B. W. Strijdom: Effect of herbicides on survival of rhizobia and nodulation of peas, groundnuts and lucerne. S. Afric. J. Plant Soil 1, 135-138 (1984)

[54] J. L. Hilton; J. S. Ard; L. L. Jansf.n; W. A. Gentner: The pantothenate-synthesizing enzyme. A metabolic site in the herbicidal action of chlorinated aliphatic acids. Weeds 7, 381-396 (1959)

[55] R. R. OGLE; G. F. WARREN: Fate and activity of herbicides in soils. Weeds 3, 257-273 (1954)

[56] P. N. P. CHow: Adsorption and dissipation of TCA by wheat and oats. Weed Sci. 18, $492-496$ (1970)

[57] W. E. Coleman; J. W. Munch; W. H. Kaylor; R. P. StrelCHER; H. P. Ringhand; J. R. MeIER: Gaschromatography/mass spectroscopy analysis of mutagenic extracts of aqueous chlorinated humic acid. A comparison of the byproduct to drinking water contaminants. Environ. Sci. Technol. 18, $674-681$ (1984)

[58] U. Lahl; B. STAChel; W. SChröer; B. Zeschmar: Bestimmung halogenorganischer Säuren in Wasserproben. Z. Wasser Abwasser Forsch. 17, 45-49 (1984)

[59] G. Müller; M. Spassovski; D. Henschler: Metabolism of trichloroethylene in man. Arch. Toxicol. 32, $283-295$ (1974) 